\title{
Comunicação
}

[Communication]

\section{Nasonia vitripennis (Walker) (Hymenoptera: Pteromalidae) parasitóide de dípteros muscóides coletado em Itumbiara, Goiás}

\author{
[Nasonia vitripennis (Walker) (Hymenoptera: Pteromalidae) parasitoid of muscoids dipterous collected in Itumbiara, \\ Goias, Brazil] \\ C.H. Marchiori \\ Instituto Luterano de Ensino Superior de Itumbiara-ILES-ULBRA \\ Caixa Postal 23-T \\ 75500-000 - Itumbiara, GO
}

A família Pteromalidae (Hymenoptera: Chalcidoidea) inclui grande número de espécies parasitóides, muitas de importância no controle biológico de muscóides sinantrópicos (Cardoso, Milward-De-Azevedo, 1996). Podem ser solitários ou gregários, ectoparasitóides ou endoparasitóides, parasitóides primários ou secundários, coinobiontes ou idiobiontes. A maioria é idiobionte e muitos desenvolvem-se como ectoparasitóides em larvas ou pupários de Diptera, Coleoptera, Hymenoptera, Lepidoptera e Siphonaptera (Ioriatti, 1995).

Nasonia vitripennis (Hymenoptera: Pteromalidae) comporta-se como parasitóide gregário, é ectoparasitóide em pupas de várias espécies de famílias de Diptera, particularmente de Calliphoridae, Muscidae, Sarcophagidae e Tachinidae (Rivers, Denlinger, 1995). É um inseto polífago que parasita mais de 68 espécies de dípteros (Whiting, 1967). No Brasil, Madeira e Neves (1985) relataram pela primeira vez $N$. vitripennis em pupas de Chrysomya sp.
O objetivo deste trabalho foi relatar a ocorrência do parasitóide Nasonia vitripennis parasitando pupas de dípteros muscóides em diferentes substratos em Itumbiara, Sul de Goiás, Brasil.

O estudo foi realizado no período de maio de 1998 a abril de 2002, no município de ItumbiaraGO $\left(18^{\circ} 25^{\prime} \mathrm{S}-49^{\circ} 13^{\prime} \mathrm{W}\right)$. A coleta de adultos foi realizada segundo descrição feita por Marchiori et al. (2000).

A prevalência de parasitismo foi calculada segundo Margolis et al. (1982) e Bush et al. (1997). A preferência do parasitóide Nasonia vitripennis por seus hospedeiros foi medida pelo qui-quadrado $(\mathrm{P}<0,05)$.

Coletaram-se 737 espécimens do parasitóide (Hymenoptera: Pteromalidae) em 2.048 pupas de Diptera. A prevalência de parasitismo observada foi de 2,8\% (57/2048) (Tab. 1).

Recebido para publicação em 14 de maio de 2003

Recebido para publicação, após modificações, em 29 de julho de 2003

E-mail: pesquisa.itb@ulbra.br 
Nasonia vitripennis...

Tabela 1. Nasonia vitripennis e seus hospedeiros, coletados em diferentes substratos, no período de maio de 1998 a abril de 2002 em Itumbiara, Sul de Goiás, Brasil

\begin{tabular}{lcccc}
\hline Isca/Hospedeiro & $\begin{array}{c}\text { Número total de } \\
\text { pupas coletadas }\end{array}$ & $\begin{array}{c}\text { Número de indivíduos } \\
\text { de Nasonia vitripennis }\end{array}$ & $\begin{array}{c}\text { Número de pupas } \\
\text { parasitadas }\end{array}$ & $\begin{array}{c}\text { Prevalência } \\
(\%)\end{array}$ \\
\hline Rins de bovino & & & & \\
$\quad$ Chrysomya albiceps & 420 & 276 & 12 & 2,9 \\
Vísceras de galinha & 575 & 54 & 1 & 0,6 \\
Musca domestica & 158 & 5 & 2 & 6,1 \\
Oxysarcodexia thornax & 33 & 25 & 2 & 0,5 \\
Peckia chrysostoma & 384 & 24 & & 0,9 \\
Fezes humanas & 148 & 13 & 1 & 4,8 \\
Peckia chrysostoma & 106 & 05 & 2 & 7,0 \\
Sarcodexia lambens & 42 & 08 & 3 & 3,5 \\
Peixe & 128 & 56 & 3 & 4,0 \\
Chrysomya albiceps & 43 & 40 & & 46,2 \\
Peckia chrysostoma & 85 & 16 & 20 & 2,6 \\
Fígado & 777 & 338 & 6 & 0,9 \\
Chrysomya albiceps & 501 & 184 & 4 & \\
Chrysomya megacephala & 130 & 115 & 1 & \\
Oxysarcodexia thornax & 154 & 32 & & \\
Synthesiomyia nudiseta & 109 & 07 & & \\
\hline
\end{tabular}

O fígado foi o substrato que atraiu maior número de exemplares de $N$. vitripennis com $45,9 \%$ dos indivíduos coletados e apresentou, também, maior diversidade de espécies de dípteros muscóides parasitadas $(50,0 \%)$. Chrysomya megacephala (Fabricius) (Diptera: Calliphoridae) foi a espécie mais parasitada pelo parasitóide N. vitripennis, apresentando prevalência de parasitismo de 46,2\% (Tab. 1).

Em rins de bovinos, $N$. vitripennis apresentou preferência por $C$. albiceps. No substrato frango, $N$. vitripennis apresentou preferência por Peckia chrysostoma (Wiedemann) (Diptera: Sarcophagidae) e Musca domestica L. (Diptera: Muscidae). Em peixe, N. vitripennis, apresentou preferência por C. albiceps e P. chrysostoma. Em fezes humanas, $N$. vitripennis. apresentou preferência por pupas de $P$. chrysostoma e Sarcodexia lambens (Walker) (Diptera: Sarcophagidae). Em fígado, $N$. vitripennis apresentou preferência por $C$. albiceps, Oxysarcodexia thornax (Walker) (Diptera: Sarcophagidae) e Synthesiomyia nudiseta (Wulp) (Diptera: Muscidae) $\quad\left(\lambda^{2}=2764,2 ; \quad G L=24\right.$; $\mathrm{P}<0,0001)$.

Este trabalho relata a primeira ocorrência de $N$. vitripennis parasitando pupas de Sarcodexia lambens no Brasil.

Palavras-chave: inimigo natural, Hymenoptera, Diptera, moscas

\begin{abstract}
This work reports the occurrence of parasitoid Nasonia vitripennis (Walker) (Hymenoptera: Pteromalidae) parasitizing pupae of flies (Diptera) in different substrata in Itumbiara, Goiás. The pupae were obtained by the flotation method. They were individually placed in gelatin capsules until the emergence of flies or their parasitoids. From May 1998 through April 2002, 737 parasitoids were collected in human feces, cattle liver, chicken, fish and cattle kidney. The prevalence of parasitism was $67.8 \%$.
\end{abstract}

Keywords: natural enemy, Hymenoptera, Diptera, flies 


\section{REFERÊNCIAS BIBLIOGRÁFICAS}

BUSH, A.O.; LAFFERTY, K.D.; LOTZ , J.M. et al. Parasitology meets ecology on its own terms: Margolis et al. Revisited. J. Parasitol., v.84, p.575-583, 1997.

CARDOSO, D.; MILWARD-DE-AZEVEDO, E. Aspectos da biologia de Nasonia vitripennis (Walker), (Hymenoptera, Pteromalidae) em pupas de Chrysomya megacephala (Fabricius) e C. albiceps (Wiedemann) (Diptera, Calliphoridae), sob condições de laboratório. Rev. Bras. Entomol., v.40, p.143-146, 1996.

IORIATTI, M.C.S.S. Contribuição ao estudo da biologia e taxonomia dos Hymenoptera parasitóides de Diptera das famílias Tephritidae e Lonchaeidae. 1995. 92f. Tese (Doutorado). Universidade Federal de São Carlos, São Carlos, SP.

MADEIRA, N.G.; NEVES, D.P. Encontro de microhimenópteros Spalangia endius e Nasonia vitripennis (Pteromalidae) em pupas de
Calliphoridae (Diptera) em Belo Horizonte (MG). In: CONGRESSO BRASILEIRO DE ZOOLOGIA, 1985, Campinas. Anais... Campinas, 1985. p.388-339.

MARCHIORI, C.H.; CASTRO, M.E.V. et al. Dípteros muscóides de importância médica e veterinária e seus parasitóides em Goiás. Arq. Bras. Med. Vet. Zootec., v.52, p.350-353, 2000.

MARGOLIS, L.; ESCH, G.W.; HOLMES, J.C. et al. The use of ecological terms in parasitology (report of a ad hoc committee of the American Society of Parasitologists. J. Parasitol., v.68, p.131-133, 1982.

RIVERS, D.B.; DENLINGER, D.L. Fecundity and development of the ectoparasitic wasp Nasonia vitripennis are dependent on host quality. Entomol. Exp. Appl., v.76, p.15-24, 1995.

WHITING, A.R. The biology of the parasitic wasp Mormoniella vitripennis [Nasonia brevicornis] (Walker). Q. Rev. Biol., v.42, p.333406, 1967. 